Mon. Not. R. Astron. Soc. 000,110(2012) Printed 24 September $2018 \quad$ (MN LATEX style file v2.2)

\title{
Nuclear Star Clusters and the Stellar Spheroids of their Host Galaxies
}

\author{
Nathan Leigh ${ }^{1 \star}$, Torsten Böker ${ }^{1}$, Christian Knigge ${ }^{2}$ \\ ${ }^{1}$ European Space Agency, Space Science Department, Keplerlaan 1, 2200 AG Noordwijk, The Netherlands \\ ${ }^{2}$ School of Physics and Astronomy, University of Southampton, Highfield, Southampton, SO17 1BJ, United Kingdom
}

24 September 2018

\begin{abstract}
We combine published photometry for the nuclear star clusters and stellar spheroids of 51 low-mass, early-type galaxies in the Virgo cluster with empirical mass-to-light ratios, in order to complement previous studies that explore the dependence of nuclear star cluster (NSC) masses on various properties of their host galaxies. We confirm a roughly linear relationship between NSC mass and luminous host spheroid mass, albeit with considerable scatter $(0.57 \mathrm{dex})$. In order to translate this to an $M_{\mathrm{NSC}}-\sigma$ relation, we estimate velocity dispersions from the virial theorem, assuming all galaxies in our sample share a common dark matter fraction and are dynamically relaxed. We then find that $M_{\mathrm{NSC}} \sim \sigma^{2.73 \pm 0.29}$, with a slightly reduced scatter of 0.54 dex.

This confirms recent results that the shape of the $M_{\mathrm{CMO}}-\sigma$ relation is different for NSCs and super-massive black holes (SMBHs). We discuss this result in the context of the generalized idea of "central massive objects" (CMOs).

In order to assess which physical parameters drive the observed nuclear cluster masses, we also carry out a joint multi-variate power-law fit to the data. In this, we allow the nuclear cluster mass to depend on spheroid mass and radius (and hence implicitly on velocity dispersion), as well as on the size of the globular cluster reservoir. When considered together, the dependences on $M_{\mathrm{Sph}}$ and $R_{\mathrm{Sph}}$ are roughly consistent with the virial theorem, and therefore $M_{\mathrm{NSC}} \propto \sigma^{2}$. However, the only statistically significant correlation appears to be a simple linear scaling between NSC mass and luminous spheroid mass.

We proceed to directly compare the derived NSC masses with predictions for two popular models for NSC formation, namely i) globular cluster infall due to dynamical friction, and ii) in-situ formation during the early phases of galaxy formation that is regulated via momentum feedback from stellar winds and/or supernovae. Neither model can directly predict the observations, and we discuss possible interpretations of our findings.
\end{abstract}

Key words: galaxies: nuclei - galaxies: elliptical and lenticular, cD - galaxies: photometry - methods: statistical

\section{INTRODUCTION}

The presence of a central massive object in the nuclei of galaxies appears to be a generic by-product of their formation and evolution. Over the last decade, it has become clear that in galaxies with massive stellar spheroids (i.e. massive ellipticals or early-type spirals with bulges more massive than $\gtrsim 10^{10} \mathrm{M}_{\odot}$ ), the nucleus is nearly always occupied by a super-massive black hole that outweighs by far the stellar mass within the central few parsecs. On the other hand, in most galaxies without massive stellar

* E-mail: nleigh@rssd.esa.int spheroids, such as disk-dominated spirals and low-mass ellipticals, an SMBH is only rarely present (Satvapal et al. 2009). Instead, the central mass concentration is dominated by a compact and massive nuclear star cluster. In between the two regimes, there appears to be a "transitional" spheroid mass range $\left(8 \lesssim \log \left(M_{\mathrm{Sph}}\left[\mathrm{M}_{\odot}\right]\right) \lesssim 10\right)$ over which the two CMO types can co-exist, and have comparable masses (Graham \& Spitler 2009; Neumaver \& Walcher 2012).

It is still unclear, however, whether these two incarnations of CMOs form through different physics, or are merely different evolutionary stages of a common formation mechanism. This question has triggered a very active area of astrophysical research, with many 
observational studies of the statistics and properties of NSCs (Carollo, Stiavelli \& Mack 1998; Böker et al. 2002, 2004; Balcells et al. 2003; Graham \& Guzman 2003; Walcher et al. 2005, 2006; Cote et al. 2006) and SMBHs (Satvapal et al. 2008; Gallo et al. 2010) in lowmass spheroids, their co-existence (Graham \& Driver 2007; Seth et al. 2008; Gonzalez Delgado et al. 2008), as well as a number of proposed theories about the growth of NSCs (Capuzzo-Dolcetta \& Miocchi 2008; Agarwal \& Milosavlievic 2011; Hartmann et al. 2011; Antonini et al. 2012) and the mutual feedback between NSC and SMBH (McLaughlin, King \& Nayakshin 2006; Navakshin. Wilkinson \& King 2009).

In 2006, three independent studies (Wehner \& Harris 2006; Rossa et al. 2006; Ferrarese et al. 2006a) pointed out that NSC masses appear to correlate with the masses of their host spheroids, confirming an earlier result by Graham \& Guzman (2003). These scaling relations are similar to those observed for SMBHs (Ferrarese \& Merritt 2000; Gebhardt et al. 2000; Häring \& Rix 2004), i.e. $M_{\mathrm{CMO}} \propto \sigma^{\beta}$. However, because of the observational difficulties to obtain reliable NSC masses, and the relatively small range of spheroid masses over which NSCs dominate over SMBHs, it is still debated whether both types of CMOs follow the same scaling relation (i.e. have the same value of $\beta$ ), or whether they depend in different ways on the properties of their host spheroid.

While Ferrarese et al. 2006a) suggested that NSCs smoothly extend the SMBH relations to lower masses, with $\beta \approx 4$ in both cases, a recent study by Graham (2012) concluded that the two types of CMOs depend differently on the total spheroid mass: NSCs follow a relation with $\beta=1.57 \pm 0.24$ while for SMBHs, $4 \lesssim \beta \lesssim 5$.

In order to add to the current discussion, we reexamine photometry of the stellar spheroids and NSCs in 51 early-type galaxies, obtained with the Hubble Space Telescope ACS Virgo Cluster Survey, and published in Ferrarese et al. (2006b) and Cote et al. (2006). The analysis of Ferrarese et al. (2006a) compared the luminous mass of 29 NSCs to the total mass of their host galaxies, the latter being estimated from stellar velocity dispersion measurements. Here, we follow a somewhat different approach by comparing the luminous mass of both NSC and galaxy spheroids, derived via color-corrected mass-to-light ratios. This not only yields more reliable stellar masses, but also improves the statistics by increasing our sample size to 51 since we can use nearly the entire sample of NSCs identified in Cote et al. (2006).

This paper is structured as follows. In $\S 2$ we summarize the data sources for our analysis and describe our method to convert the published photometry into estimates of the stellar mass of both NSCs and host spheroids. We present a quantitative analysis of the resulting $M_{\mathrm{NSC}}-M_{\mathrm{Sph}}$ relation in $\S 3$, as well as of the inferred $M_{\mathrm{NSC}}-\sigma$ relation. In addition, we demonstrate through a multi-variate analysis that the only statistically significant correlation with any observed parameter is a simple linear scaling of NSC mass with spheroid mass. In $\S$, we briefly summarize the theoretical predictions for two models of NSC formation, compare the present-day NSC masses to those predictions, and discuss the implications for the plausibility of either model. We conclude with a discussion and summary of our results and the implications for the origin of NSCs in $\S 5$ and $\S 6$. respectively.

\section{THE DATA}

For our analysis, we use data for 51 nucleated early-type galaxies observed during the Advanced Camera for Surveys Virgo Cluster Survey (ACSVCS, Cote et al. 2004). Taking advantage of the high spatial resolution of the Hubble Space Telescope, this data set provides detailed and accurate photometry for both the galaxy bodies and their NSCs. We reject five galaxies from the original sample of Cote et al. $(2006)$ in which the apparent NSCs are significantly offset from the galaxy's photocentre and therefore, as discussed by Cote et al. (2006), may well be globular clusters that only appear to reside close to the nucleus due to a chance projection.

We note here that, given the morphological types of the sample galaxies (E, S0, dE, dS0, and dE,N), their stellar spheroids can be expected to be virialized, and to have little or no current star formation activity. This justifies use of a single (color-dependent) mass-to-light ratio for each galaxy spheroid in order to derive its stellar mass.

We make use of the apparent z-band magnitudes, (g-z) colors, and half-light radii for both NSCs (from Cote et al. 2006) and host spheroids (from Ferrarese et al. 2006b). In order to convert to absolute magnitudes and physical radii, we follow the approach of Cote et al. (2006) and assume a common distance of $16.5 \mathrm{Mpc}$ for all galaxies. For convenience, the observed galaxy and NSC magnitudes are listed again in Columns 2 and 3 of Table 1. We also list in Column 4 the (completeness-corrected) total number of globular clusters (GCs) as provided in Peng et al. (2008, their Table 2) which we will use in $\S 4.2$.

In order to obtain estimates for the stellar masses of both NSCs and spheroids, we need to multiply their respective z-band luminosities by an appropriate mass-to-light ratio. We use the empirically calibrated mass-to-light ratios provided by Bell et al. (2003), accounting for the (g-z) color of NSC and spheroid, respectively, and list the resulting values for $M_{\mathrm{Sph}}$ and $M_{\mathrm{NSC}}$ in Columns 5 and 8 of Table 1 . We also provide estimates for the average GC mass $\bar{m}_{\mathrm{GC}}$ for our sample of galaxies. This is done by multiplying the z-band galaxy masses by the percentage of the total mass of the galaxy in GCs (obtained in the z-band) taken from Peng et al. (2008). This gives an estimate for the total mass of the GC system, which we then divide by the total number of GCs in order to arrive at an estimate for the average GC mass. Note that some of the percentages provided in Peng et al. (2008) are negative. In these few cases, we leave blank the corresponding entry for $\bar{m}_{\mathrm{GC}}$ in Table 1 .

\section{$3 \quad M_{\mathrm{NSC}}$ SCALING RELATIONS}

In this section, we present a quantitative analysis of the observed $M_{\mathrm{NSC}}-M_{\mathrm{Sph}}$ relation using estimates for luminous masses derived from photometry. We also consider the inferred $M_{\mathrm{NSC}}-\sigma$ relation found by assuming virial equilibrium, in addition to a multi-variate analysis that quantifies 
Table 1. Properties of the sample galaxies, identified by their VCS number (Column 1). Columns 2 and 3 list the absolute z-band magnitudes of the galaxy (Ferrarese et al. 2006b) and NSC (Cote et al. 2006) (after accounting for a distance of 16.5 Mpc to get the absolute magnitude), respectively. Column 4 provides the completeness-corrected number of GCs $\left(N_{\mathrm{GC}}\right)$ (Peng et al. 2008). Column 5 provides the total stellar mass of the galaxy $M_{\text {Sph }}$ calculated from its z-band magnitude; in units of $10^{9} \mathrm{M}_{\odot}$. Column 6 gives the effective radius $R_{\mathrm{Sph}}$ of the galaxy spheroid (Ferrarese et al. 2006b). Column 7 lists the calculated velocity dispersion within the effective radius of the galaxy in $\mathrm{km} \mathrm{s}^{-1}$. Column 8 gives the inferred stellar mass of the NSC $M_{\mathrm{NSC}}$, column 9 its half-light radius (Cote et al. 2006), and column 10 its calculated velocity dispersion. Finally, Column 11 gives the average globular cluster mass $\bar{m}_{\mathrm{GC}}$ in units of $10^{5} \mathrm{M}_{\odot}$. All listed errors denote the $1 \sigma$ uncertainties.

\begin{tabular}{|c|c|c|c|c|c|c|c|c|c|c|}
\hline $\begin{array}{l}\text { VCS } \\
(1)\end{array}$ & $\begin{array}{c}M_{z} \\
\text { (Galaxy) } \\
(2)\end{array}$ & $\begin{array}{c}M_{z} \\
(\mathrm{NSC}) \\
(3)\end{array}$ & $\begin{array}{c}N_{\mathrm{GC}} \\
(4)\end{array}$ & $\begin{array}{c}M_{\mathrm{Sph}} \\
\left(10^{9} \mathrm{M}_{\odot}\right) \\
(5)\end{array}$ & $\begin{array}{c}R_{\mathrm{Sph}} \\
(\mathrm{kpc}) \\
(6)\end{array}$ & $\begin{array}{c}\sigma \\
\left(\mathrm{km} \mathrm{s}^{-1}\right) \\
(7)\end{array}$ & $\begin{array}{c}M_{\mathrm{NSC}} \\
\left(10^{5} \mathrm{M}_{\odot}\right) \\
(8)\end{array}$ & $\begin{array}{c}R_{\mathrm{NSC}} \\
(\mathrm{pc}) \\
(9)\end{array}$ & $\begin{array}{c}\sigma_{\mathrm{NC}} \\
\left(\mathrm{km} \mathrm{s}^{-1}\right) \\
(10)\end{array}$ & $\begin{array}{c}\bar{m}_{\mathrm{GC}} \\
\left(10^{5} \mathrm{M}_{\odot}\right) \\
(11)\end{array}$ \\
\hline 27 & -20.68 & -14.27 & $62.0 \pm 13.0$ & 23.59 & 2.18 & 241.19 & $708.86 \pm 53.55$ & 6.81 & $236.61 \pm 0.81$ & 4.57 \\
\hline 29 & -20.19 & -15.51 & $84.0 \pm 19.0$ & 16.08 & 0.80 & 328.87 & $2188.37 \pm 165.32$ & 25.87 & $213.26 \pm 1.03$ & 2.68 \\
\hline 30 & -20.73 & -13.49 & $83.0 \pm 25.0$ & 21.78 & 2.00 & 241.74 & $251.25 \pm 18.98$ & 1.92 & $265.09 \pm 28.65$ & 4.20 \\
\hline 31 & -20.29 & -12.95 & $116.0 \pm 24.0$ & 17.83 & 1.34 & 267.95 & $231.43 \pm 17.48$ & 2.80 & $210.68 \pm 13.11$ & 4.76 \\
\hline 32 & -20.26 & -14.41 & $50.0 \pm 14.0$ & 16.81 & 1.09 & 287.28 & $862.06 \pm 65.12$ & 12.89 & $189.59 \pm 3.04$ & 2.35 \\
\hline 36 & -20.0 & -14.13 & $69.5 \pm 9.8$ & 11.21 & 0.84 & 267.70 & $623.10 \pm 47.07$ & 16.66 & $141.81 \pm 2.97$ & - \\
\hline 37 & -20.10 & -12.90 & $20.1 \pm 7.3$ & 9.88 & 0.94 & 237.25 & $197.75 \pm 14.94$ & 2.08 & $225.96 \pm 21.88$ & 2.95 \\
\hline 38 & -20.06 & -15.37 & $47.0 \pm 11.0$ & 13.93 & 1.05 & 267.09 & $2118.26 \pm 160.02$ & 40.13 & $168.47 \pm 1.03$ & 3.56 \\
\hline 39 & -19.64 & -17.14 & $72.0 \pm 12.0$ & 8.45 & 1.29 & 188.07 & $8197.84 \pm 673.67$ & 62.47 & $277.04 \pm 1.03$ & 4.58 \\
\hline 42 & -19.74 & -15.14 & $71.0 \pm 14.0$ & 8.74 & 1.15 & 201.91 & $1615.18 \pm 122.01$ & 47.81 & $134.77 \pm 4.30$ & 3.82 \\
\hline 44 & -19.44 & -11.58 & $52.3 \pm 8.5$ & 7.15 & 0.89 & 207.37 & $38.14 \pm 2.88$ & 4.81 & $65.32 \pm 1.34$ & 2.19 \\
\hline 47 & -19.70 & -12.02 & $58.6 \pm 9.3$ & 8.60 & 1.56 & 172.21 & $89.90 \pm 6.79$ & 4.24 & $106.71 \pm 3.02$ & 3.23 \\
\hline 48 & -19.21 & -12.81 & $35.1 \pm 7.6$ & 4.09 & 1.61 & 116.75 & $142.51 \pm 10.77$ & 2.88 & $163.02 \pm 9.69$ & 3.38 \\
\hline 49 & -19.71 & -12.48 & $114.0 \pm 12.0$ & 6.94 & 1.22 & 175.08 & $113.26 \pm 8.56$ & 3.28 & $136.17 \pm 6.48$ & 3.41 \\
\hline 50 & -18.73 & -12.09 & $24.9 \pm 6.0$ & 3.85 & 1.76 & 108.50 & $73.43 \pm 5.55$ & 2.80 & $118.67 \pm 7.38$ & 3.25 \\
\hline 51 & -18.57 & -10.79 & $17.2 \pm 5.4$ & 2.77 & 1.01 & 121.20 & $21.05 \pm 1.59$ & 2.48 & $67.52 \pm 5.07$ & 3.06 \\
\hline 52 & -18.32 & -13.61 & $10.4 \pm 5.0$ & 2.94 & 0.55 & 168.80 & $304.45 \pm 23.00$ & 8.65 & $137.56 \pm 0.74$ & 5.93 \\
\hline 55 & -18.33 & -12.46 & $48.7 \pm 8.4$ & 2.67 & 0.96 & 122.24 & $100.97 \pm 7.63$ & 3.04 & $133.56 \pm 7.26$ & 5.98 \\
\hline 57 & -18.25 & -13.24 & $43.4 \pm 7.9$ & 2.00 & 1.34 & 89.75 & $196.63 \pm 14.85$ & 12.25 & $92.88 \pm 1.38$ & 2.54 \\
\hline 58 & -18.27 & -9.90 & $21.3 \pm 6.1$ & 2.10 & 0.74 & 123.27 & $7.76 \pm 0.59$ & 1.76 & $48.67 \pm 5.90$ & 1.77 \\
\hline 59 & -18.16 & -11.02 & $10.8 \pm 5.6$ & 1.77 & 2.44 & 62.41 & $23.81 \pm 1.80$ & 3.04 & $64.85 \pm 3.52$ & 0.82 \\
\hline 60 & -18.64 & -12.20 & $66.0 \pm 9.5$ & 3.05 & 1.63 & 100.27 & $88.16 \pm 6.66$ & 2.16 & $148.05 \pm 13.60$ & 4.02 \\
\hline 62 & -18.34 & -12.03 & $37.6 \pm 7.4$ & 2.54 & 1.46 & 96.74 & $60.80 \pm 4.59$ & 9.53 & $58.56 \pm 0.49$ & 4.67 \\
\hline 63 & -18.19 & -9.89 & $18.1 \pm 5.5$ & 2.19 & 1.46 & 89.64 & $10.74 \pm 0.81$ & 15.70 & $19.18 \pm 0.38$ & 1.81 \\
\hline 64 & -18.25 & -12.55 & $60.6 \pm 9.3$ & 2.43 & 0.79 & 128.46 & $104.93 \pm 7.93$ & 18.66 & $54.98 \pm 1.25$ & 3.13 \\
\hline 65 & -18.04 & -9.78 & $40.7 \pm 7.6$ & 1.84 & 0.79 & 111.76 & $7.21 \pm 0.54$ & 1.44 & $51.87 \pm 8.13$ & 2.98 \\
\hline 66 & -18.32 & -9.86 & $14.4 \pm 5.7$ & 2.05 & 2.17 & 71.29 & $10.60 \pm 0.80$ & 1.92 & $54.46 \pm 5.88$ & 1.28 \\
\hline 68 & -18.69 & -12.08 & $42.1 \pm 7.9$ & 2.96 & 2.08 & 87.38 & $61.80 \pm 4.67$ & 6.65 & $70.70 \pm 0.31$ & 3.23 \\
\hline 69 & -18.18 & -11.89 & $23.9 \pm 6.1$ & 1.79 & 1.53 & 79.34 & $56.71 \pm 4.28$ & 2.32 & $114.57 \pm 9.50$ & 5.38 \\
\hline 70 & -17.01 & -9.82 & $2.2 \pm 4.2$ & 0.72 & 0.73 & 72.46 & $7.21 \pm 0.54$ & 2.56 & $38.90 \pm 2.79$ & - \\
\hline 71 & -17.80 & -9.15 & $15.5 \pm 5.8$ & 1.10 & 1.05 & 75.13 & $3.92 \pm 0.30$ & 3.04 & $26.31 \pm 1.43$ & 0.36 \\
\hline 74 & -17.34 & -8.10 & $7.3 \pm 4.2$ & 0.70 & 0.84 & 66.84 & $1.28 \pm 0.10$ & 2.00 & $18.57 \pm 1.90$ & 0.29 \\
\hline 76 & -17.29 & -8.48 & $6.3 \pm 4.3$ & 0.87 & 0.84 & 74.74 & $2.05 \pm 0.16$ & 1.68 & $25.62 \pm 3.30$ & 0.96 \\
\hline 78 & -17.74 & -10.21 & $54.2 \pm 8.8$ & 1.33 & 0.86 & 91.24 & $11.46 \pm 0.87$ & 2.96 & $45.59 \pm 2.59$ & 2.51 \\
\hline 79 & -18.14 & -13.19 & $-6.0 \pm 13.0$ & 2.35 & 0.57 & 149.06 & $197.79 \pm 14.94$ & 9.69 & $104.75 \pm 0.93$ & - \\
\hline 81 & -17.55 & -10.98 & $16.5 \pm 5.2$ & 1.11 & 1.43 & 64.56 & $21.78 \pm 1.65$ & 3.12 & $61.23 \pm 3.18$ & 2.21 \\
\hline 83 & -17.39 & -13.70 & $3.6 \pm 3.7$ & 1.19 & 0.31 & 143.46 & $380.80 \pm 28.77$ & 15.78 & $113.91 \pm 2.28$ & 39.88 \\
\hline 84 & -17.66 & -12.71 & $26.7 \pm 6.8$ & 1.31 & 0.62 & 107.05 & $139.98 \pm 10.57$ & 4.49 & $129.54 \pm 3.20$ & 1.43 \\
\hline 85 & -16.96 & -11.87 & $28.7 \pm 6.7$ & 0.52 & 0.73 & 61.78 & $54.45 \pm 4.11$ & 2.64 & $105.24 \pm 7.19$ & 9.82 \\
\hline 86 & -17.15 & -9.68 & $9.2 \pm 4.3$ & 0.78 & 0.94 & 66.97 & $6.63 \pm 0.50$ & 5.29 & $25.96 \pm 0.40$ & 1.79 \\
\hline 88 & -17.92 & -10.92 & $9.2 \pm 4.3$ & 1.79 & 1.04 & 96.26 & $22.04 \pm 1.66$ & 2.80 & $65.01 \pm 4.05$ & 2.53 \\
\hline 89 & -17.47 & -10.59 & $20.4 \pm 5.8$ & 0.97 & 1.32 & 62.89 & $15.67 \pm 1.18$ & 4.57 & $42.96 \pm 1.02$ & 4.52 \\
\hline 90 & -17.10 & -10.94 & $0.6 \pm 3.5$ & 0.36 & 0.39 & 70.05 & $23.82 \pm 1.80$ & 2.40 & $73.00 \pm 5.76$ & - \\
\hline 91 & -17.43 & -11.69 & $49.7 \pm 8.6$ & 0.94 & 0.87 & 76.12 & $42.52 \pm 3.21$ & 10.17 & $47.41 \pm 0.48$ & 1.30 \\
\hline 92 & -16.93 & -10.05 & $3.9 \pm 2.6$ & 0.51 & 1.07 & 50.75 & $9.60 \pm 0.73$ & 3.28 & $39.64 \pm 1.89$ & 3.82 \\
\hline 93 & -16.94 & -12.70 & $-9.0 \pm 14.0$ & 0.80 & 0.27 & 127.22 & $142.87 \pm 10.79$ & 5.05 & $123.38 \pm 2.19$ & - \\
\hline 95 & -17.12 & -11.28 & $31.0 \pm 7.0$ & 0.69 & 1.79 & 45.48 & $32.10 \pm 2.42$ & 21.22 & $28.51 \pm 0.70$ & 5.36 \\
\hline 96 & -17.37 & -11.18 & $14.0 \pm 5.7$ & 0.91 & 1.69 & 53.86 & $26.00 \pm 1.96$ & 4.00 & $59.08 \pm 1.90$ & 1.31 \\
\hline 97 & -16.71 & -12.18 & $4.5 \pm 3.9$ & 0.50 & 0.55 & 69.86 & $78.02 \pm 5.89$ & 2.00 & $144.74 \pm 14.80$ & 0.89 \\
\hline 99 & -16.41 & -9.58 & $11.7 \pm 4.8$ & 0.34 & 0.89 & 45.22 & $5.61 \pm 0.42$ & 4.65 & $25.49 \pm 0.58$ & 1.33 \\
\hline 100 & -17.40 & -11.84 & $10.2 \pm 4.7$ & 1.08 & 3.15 & 42.96 & $51.42 \pm 3.88$ & 6.57 & $64.88 \pm 0.32$ & 0.85 \\
\hline
\end{tabular}


the dependence of $M_{\mathrm{NSC}}$ on the individual galaxy parameters. In all cases, the fits are normalized by dividing the parameters being compared by their sample-averaged values. This significantly reduces the uncertainties in the offsets (i.e. the y-intercepts for our lines of best-fit). All uncertainties given in this paper correspond to the $1 \sigma$-confidence level.

\subsection{The $M_{\mathrm{NSC}}-M_{\mathrm{Sph}}$ correlation}

Figure 1 plots the stellar mass of the NSCs against that of their host spheroids. The solid line shows a weighted leastsquares fit to the data found using a relation of the form

$$
\log \left(M_{\mathrm{NSC}} / 10^{7.6} \mathrm{M}_{\odot}\right)=a+b \cdot \log \left(M_{\mathrm{Sph}} / 10^{9.7} \mathrm{M}_{\odot}\right) .
$$

The uncertainties were calculated via a bootstrap methodology (Leigh. Sills \& Knigge 2011) in which we generated 1,000 fake data sets by randomly sampling (with replacement) data points from the observations. We obtained lines of best fit for each fake data set, fit a Gaussian to the subsequent distribution and extracted its standard deviation. We find $a=-0.53 \pm 0.09$ and $b=1.18 \pm 0.16$, with a scatter of 0.57 dex. A strong correlation between the two quantities is evident, with a Spearman correlation coefficient of $r_{s}=0.70$, a significance level at which the null hypothesis of zero correlation is disproved of $p_{s}=7.17 \cdot 10^{-9}$, and a slope that is inconsistent with zero at the $7 \sigma$ confidence level. Table 1 does not list uncertainties for the spheroid masses provided in column 5, since we did not have uncertainties for the z-band magnitudes from which they were calculated. However, we performed a doubly-weighted least-squares fit using the spheroid masses and uncertainties provided in column 8 of Table 1 in Peng et al. (2008) (who derived their masses using different assumptions for the mass-to-light ratios than adopted in this paper). The slope and y-intercept for the resulting line of best-fit are the same as found using the spheroid masses calculated in this paper to within one standard deviation.

This result is in broad agreement with various other studies. For example, using the HST imaging data of Lotz, Miller \& Ferguson (2004) for 45 nucleated dE,N galaxies in Leo, Virgo and Fornax, Wehner \& Harris (2006) find values of $a=-3.10 \pm 0.81$ and $b=1.18 \pm 0.10$. Using HST near-infrared images of early-type spiral bulges, Balcells, Graham \& Peletier (2007) find $a=-2.75 \pm 0.15$ and $b=0.76 \pm 0.13$. Our analysis, which should be an improvement over these earlier studies due to the larger sample size and the use of color-dependent mass-to-light ratios, therefore re-affirms an approximately linear dependence of $M_{\mathrm{NSC}}$ on the luminous mass of the host spheroid.

\subsection{The $M_{\mathrm{NSC}}-\sigma$ correlation}

As mentioned earlier, we assume that the sample galaxies are in dynamical equilibrium, and that, therefore, the virial theorem can be used to predict the stellar velocity dispersion of the spheroid. We use the empirically calibrated formula of Cappellari et al. (2006):

$$
\sigma^{2}=\frac{G M_{\mathrm{Sph}}}{5 f_{g} R_{\mathrm{Sph}}}
$$

where $M_{\mathrm{Sph}}$ is the luminous mass of the galaxy spheroid, $R_{\mathrm{Sph}}$ is its half-mass radius, and $f_{g}=\Omega_{b} / \Omega_{m}$ is the bary-

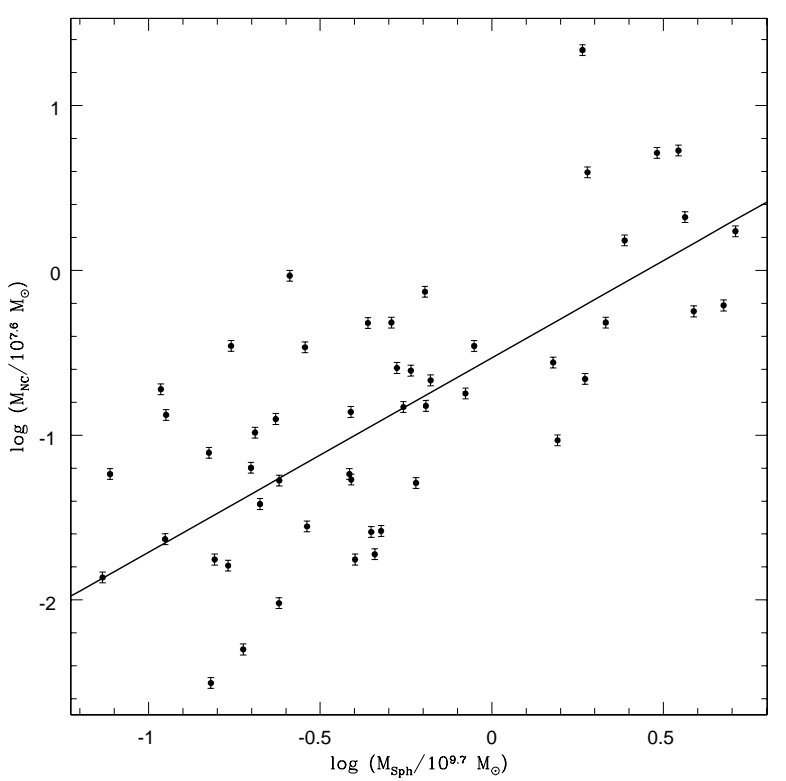

Figure 1. Correlation between the stellar mass of NSCs and their host spheroids. The solid line indicates the best-fitting linear relationship of Equation 1 Error bars were calculated using the 0.041 mag uncertainty quoted by Cote et al. (2006).

onic mass fraction. We assume that $f_{g}=0.16$ (Spergel et al. 2003) for all sample galaxies, and that the measured halflight radii (Column 6 of Table 1) are a good approximation of the true half-mass radii, i.e. that luminous and dark matter follow the same radial distribution. The resulting values for the predicted velocity dispersion are listed in Column 7 of Table 1. Figure 2 then plots the relation between $M_{\mathrm{NSC}}$ and $\sigma$. The best fit is again indicated by the solid line, it is described by

$\log \left(\frac{M_{\mathrm{NSC}}}{10^{7.6} \mathrm{M}_{\odot}}\right)=(-0.70 \pm 0.07)+(2.73 \pm 0.29) \cdot \log \left(\frac{\sigma}{128 \mathrm{~km} \mathrm{~s}^{-1}}\right)$.

This correlation is slightly stronger $\left(r_{s}=0.72\right.$ and $p_{s}=$ $1.48 \cdot 10^{-9}$ ) than that between $M_{\mathrm{Sph}}$ and $M_{\mathrm{NSC}}$, and has a slightly reduced scatter (0.54 dex).

The best-fit slope disagrees with that of Ferrarese et al. (2006a) who conclude, based on measured velocity dispersions of a smaller subset (29 objects) of the sample discussed here, that $\log \left(M_{\mathrm{NSC}}\right)=(6.91 \pm 0.11)+(4.27 \pm 0.61)$. $\log \left(\sigma / 54 \mathrm{~km} \mathrm{~s}^{-1}\right)$. Taken at face value, this disagreement implies that our above assumptions are wrong, and that either the half-light radius is a poor indicator of the half-mass radius, or that $f_{g}$ varies across the galaxy sample. For example, in order for Equation 2 to yield the slope of Ferrarese et al. (2006a), $f_{g}$ would have to scale with the square root of the luminous spheroid mass.

On the other hand, it is also possible that the relatively small sample used for the Ferrarese et al. (2006a) analysis causes systematic errors in the derived slope of the fit. This last possibility appears to be supported by a recent study of Graham (2012) who re-analyzed the Ferrarese et al. (2006a) data, together with complementary literature data, and con- 


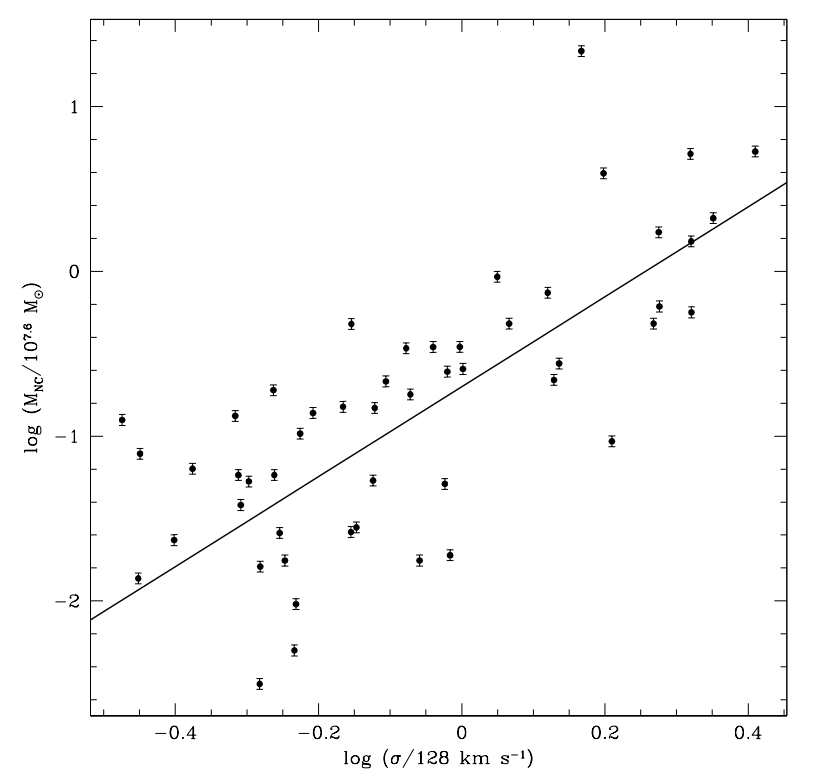

Figure 2. Correlation between the stellar mass of NSCs and the predicted velocity dispersion of their host spheroids. The solid line indicates the best-fitting linear relationship of Equation 3 Error bars are described as in Figure 1

cluded that $M_{\mathrm{NSC}} \propto \sigma^{1.57 \pm 0.24}$, in better agreement with Figure 2

\subsection{Multi-variate Analysis}

We now attempt to quantify whether or not the host galaxy properties are correlated in a way that is consistent with our use of the virial theorem as described in the previous section. More specifically, Equation 2 implies that the (total) mass of the spheroid and its half-mass radius are correlated. If the luminous matter indeed follows the same radial distribution as the dark matter, this should also be true for the luminous spheroid mass and its half-light radius $R_{\mathrm{Sph}}$.

To assess whether or not this is indeed the case, we perform a multi-variate analysis for our sample, using a generalized relation of the form

$$
M_{\mathrm{NSC}} \sim \epsilon M_{\mathrm{Sph}}^{\alpha} R_{\mathrm{Sph}}^{\beta} N_{\mathrm{GC}}^{\delta},
$$

where $\alpha, \beta, \delta$, and $\epsilon$ are all free parameters.

Note that we have included the total number of GCs, $N_{\mathrm{GC}}$, in this analysis, since the dependence of $M_{\mathrm{NSC}}$ on this parameter will be tested in $\S 4$. We perform a direct weighted least-squares fit to Equation 4 , and calculate $2 \sigma$ and $3 \sigma$ joint confidence intervals for the correlations between possible pair of parameters $\alpha, \beta$, and $\delta$. This is done by calculating the chi-squared value for every combination of each parameter pair, and identifying the minimum. We then find every combination that gives a chi-squared value that is within the specified confidence interval of the minimum in order to obtain our joint confidence intervals. For a description of a similar application of this technique, we refer the reader to Verbunt, Pooley \& Bassa (2007).

Our goal is to determine which of the parameters in
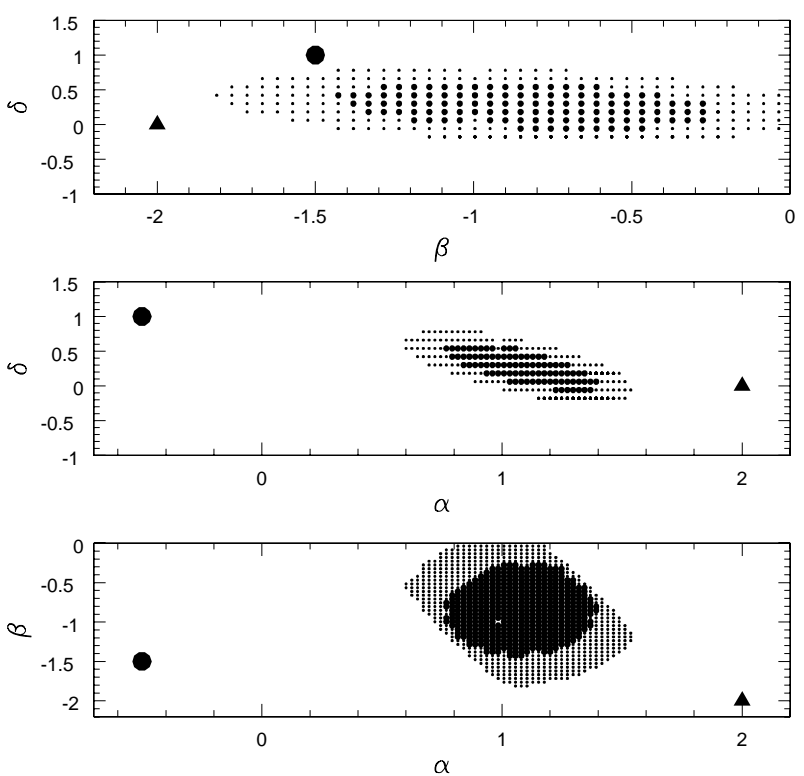

Figure 3. The large and small filled circles show the $1 \sigma$ and $2 \sigma$, respectively, joint confidence intervals on the two parameters being considered for all three projections of the 4-dimensional parameter space in Equation 4 The large solid circles and triangles show the exact power-law predictions for the GC infall and the in situ star formation models, respectively.

Table 2. Best-fitting power-law indices for the parameters of Equation 4

\begin{tabular}{cc}
\hline Parameter & Best-Fitting Value \\
\hline $\log \epsilon$ & $-1.0_{-3.0}^{+2.6}$ \\
$\alpha$ & $1.1_{-0.3}^{+0.2}$ \\
$\beta$ & $-0.9_{-0.5}^{+0.6}$ \\
$\delta$ & $0.3_{-0.4}^{+0.3}$ \\
\hline
\end{tabular}

Equation 4 are correlated with $M_{\mathrm{NSC}}$ at a statistically significant level while naturally accounting for any correlations between them. In this way, we are able to quantify the degree to which $M_{\mathrm{Sph}}$ and $R_{\mathrm{Sph}}$ are driving the correlation between $M_{\mathrm{NSC}}$ and $\sigma$.

The results are shown in Table 2 and illustrated in Figure 3 As expected given the results of $\S 3.1$ we find a roughly linear dependence of $M_{\mathrm{NSC}}$ on $M_{\mathrm{Sph}}$. If the virial theorem is valid throughout our galaxy sample, $M_{\mathrm{NSC}} \propto M_{\mathrm{Sph}}$ implies that $M_{\mathrm{NSC}} \propto R_{\mathrm{Sph}}^{-1}$. The results for $R_{\mathrm{Sph}}$ appear to be consistent with this notion, but we caution that the uncertainty for this parameter is large. Therefore, our results suggest that $M_{\mathrm{Sph}}$ is the only galaxy parameter that correlates significantly with $M_{\mathrm{NSC}}$.

\section{COMPARISON WITH PROPOSED FORMATION SCENARIOS}

In this section, we compare theoretical predictions for NSC masses from two popular formation models to the observa- 
tional data. We begin by deriving theoretical relations for each formation scenario, which we use as a basis for comparison with observational data.

\subsection{In-Situ Formation Regulated by Momentum Feedback}

A plausible mechanism for the birth of "seed" NSCs, proposed by McLaughlin. King \& Navakshin (2006), is an extension of the arguments presented in King (2003) and King (2005) for the $M_{\mathrm{SMBH}^{-}} \sigma$ relation. It involves the formation of a massive star cluster from gas accumulating at the center during the early phases of the galaxy's evolution. Very soon after the onset of star formation, stellar winds from massive stars and supernovae drive a superwind from the nucleus. Initially, the wind is momentum-conserving with a momentum flux that is proportional to the Eddington luminosity. It sweeps up the surrounding medium into a thin supershell that gets pushed outward by the ram pressure it generates. Eventually, gravity balances the ram pressure and the shell stalls. More gas then falls into and feeds the nucleus. The process is repeated until the NSC reaches a critical mass, at which point the stall radius becomes very large (since the stall radius is proportional to the mass of the NSC). This gives the gas time to cool before it can reach the stall radius, since the cooling time begins to exceed the crossing time of the bubble. The gas becomes energyconserving, and accelerates in its expansion to escape from the galaxy. This fixes the mass of the NSC at the critical mass (McLaughlin, King \& Navakshin 2006):

$$
M_{\mathrm{NSC}}=\frac{f_{g} \kappa \sigma^{4}}{\lambda \pi G^{2}}
$$

where $f_{g}=0.16$ again is the baryonic mass fraction, $\kappa=$ $0.398 \mathrm{~cm}^{2} \mathrm{~g}^{-1}$ is the electron scattering opacity, and $\sigma$ is the velocity dispersion of the spheroid. If correct, this mechanism would therefore naturally explain the $M_{\text {NSC }} \propto \sigma^{4}$ dependence suggested by Ferrarese et al. (2006a).

McLaughlin, King \& Nayakshin (2006) propose a value of $\lambda \sim 0.05$ for the efficiency of massive-star feedback from a young nuclear cluster. This is based on simple calculations for the contributions from both supernovae and stellar winds to the total momentum flux, and agrees with more detailed calculations of Leitherer, Robert \& Drissen (1992) for the total momentum deposition from solar-metallicity stars.

We can re-write Equation 5 using the virial theorem. This assumes that the present-day mass distribution of the galaxy is representative of its distribution at the time of NSC formation. In particular, if the NSC formed before the galaxy mass was fully assembled, the velocity dispersion of the surrounding spheroid may well have been lower than it is today.

Substituting the virial relation given by Equation 2 into Equation 5 this predicts for the NSC mass:

$$
M_{\mathrm{NSC}}=\frac{\kappa}{25 f_{g} \lambda \pi} M_{\mathrm{Sph}}^{2} R_{\mathrm{Sph}}^{-2} .
$$

Re-writing this in a more convenient form:

$$
M_{\mathrm{NSC}}=1.66 \times 10^{6} \mathrm{M}_{\odot}\left(\frac{1}{\lambda}\right)\left(\frac{2 \mathrm{kpc}}{R_{\mathrm{Sph}}}\right)^{2}\left(\frac{M_{\mathrm{Sph}}}{10^{9} \mathrm{M}_{\odot}}\right)^{2},
$$

where we have assumed the baryonic mass fraction $f_{g}=$ 0.16 , and the electron scattering opacity $\kappa=0.398 \mathrm{~cm}^{2} \mathrm{~g}^{-1}$.

\subsection{Infall and Merging of Globular Clusters}

The second NSC formation scenario occurs via the successive mergers of globular clusters (GCs) that spiral into the galactic centre due to dynamical friction (e.g. Tremaine, Ostriker \& Spitzer 1975; Quinlan \& Shapiro 1990). Using N-body simulations of merging GCs in galaxy nuclei, several studies have successfully reproduced some of the properties of NSCs, including their small effective radii and central velocity dispersions (e.g. Capuzzo-Dolcetta \& Miocchi 2008).

In general, the time required for a $\mathrm{GC}$ of mass $m_{\mathrm{GC}}$ that starts on a circular orbit with radius $r$ from the galactic centre to spiral into the nucleus is given by (Binney \& Tremaine 1987):

$$
\begin{aligned}
\tau_{\mathrm{dyn}}[\mathrm{yr}] & =\frac{1.65}{G \ln \Lambda} \frac{r^{2} \sigma}{m_{\mathrm{GC}}} \\
& =\frac{3.73 \times 10^{11}}{\ln \Lambda}\left(\frac{r}{2 \mathrm{kpc}}\right)^{2}\left(\frac{\sigma}{250 \mathrm{~km} \mathrm{~s}^{-1}}\right)\left(\frac{10^{6} \mathrm{M}_{\odot}}{m_{\mathrm{GC}}}\right)
\end{aligned}
$$

where $\sigma$ is the velocity dispersion of the galaxy. The Coulomb logarithm is denoted by $\ln \Lambda$, and is on the order of 10 (Binnev \& Tremaine 1987). According to Equation 8] dynamical friction operates most efficiently on the most massive GCs.

If the NSC was indeed built from GCs that have sunk into the nucleus, its mass should follow a relation of the form:

$$
M_{\mathrm{NSC}}=f_{\mathrm{GC}} M_{\mathrm{GC}} \sim f_{\mathrm{GC}} N_{\mathrm{GC}} \bar{m}_{\mathrm{GC}},
$$

where $M_{\mathrm{GC}}$ is the total mass of the globular cluster system, $N_{\mathrm{GC}}$ is the total number of GCs, $\bar{m}_{\mathrm{GC}}$ is the present-day average GC mass of the galaxy (Column 11 of Table1), and $f_{\mathrm{GC}}$ is the mass fraction of the GC system that has merged onto the NSC.

This mass fraction should be proportional to the ratio of the age of the galaxy, $\tau_{\text {gal }}$, to the time for an average GC to fall into the core due to dynamical friction, $\tau_{\text {dyn }}$ :

$$
M_{\mathrm{NSC}}=f_{\mathrm{tot}} \frac{\tau_{\mathrm{gal}}}{\tau_{\mathrm{dyn}}} N_{\mathrm{GC}} \bar{m}_{\mathrm{GC}} .
$$

Here, the additional constant $f_{\text {tot }}$ accounts for the fact that only GCs that are located within a radius at which $\tau_{\text {dyn }} \leqslant$ $\tau_{\text {gal }}$ will have had enough time to have fallen into the NSC via dynamical friction.

Substituting $\tau_{\text {dyn }}=\tau_{\text {gal }}=10$ Gyr in Equation 8 shows that for most galaxies in our sample, only GCs well inside the half-light radius $R_{\mathrm{Sph}}$ can be expected to have fallen into the nucleus. However, the present-day orbits of most GCs in our sample are comparable to or exceed the half-light radius in their host galaxies (Peng et al. 2008). In other words, most GCs will not have had sufficient time to have spiraled into the nucleus, i.e. $f_{\mathrm{GC}} \ll 1$. The compilation of Peng et al. (2008) can be used to estimate the present-day value of $f_{\text {tot }}$ by calculating the fraction of GCs inside $R_{\mathrm{Sph}}$. This yields $f_{\text {tot }} \approx 0.2$, in reasonable agreement with the notion that $N_{\mathrm{GC}}$ has not changed significantly over the lifetime of the galaxy.

We assume a universal initial GC MF that adheres to the same radial dependence in all galaxies, and use the dynamical friction timescale calculated at the half-light radius as a proxy for the corresponding volume-integrated value. 
Using Equation 8 we get from Equation 10

$$
M_{\mathrm{NSC}}=\frac{G \ln \Lambda}{1.65} \tau_{\text {gal }} \bar{m}_{G C}^{2} R_{\mathrm{Sph}}^{-2} \sigma^{-1} f_{\mathrm{tot}} N_{\mathrm{GC}},
$$

where we have used the half-light radius of the galaxy $R_{\mathrm{Sph}}$ as a proxy for the average initial orbital radius from which clusters begin spiraling into the nucleus.

Using the virial theorem to substitute for $\sigma$ (Equation 21), and $f_{\text {tot }}=0.2$ then yields

$$
M_{\mathrm{NSC}}=0.43\left(G f_{g}\right)^{1 / 2} \ln \Lambda \tau_{\mathrm{gal}} \bar{m}_{\mathrm{GC}}^{2} M_{\mathrm{Sph}}^{-1 / 2} R_{\mathrm{Sph}}^{-3 / 2} N_{\mathrm{GC}}
$$

Alternatively, we can re-write Equation 12 in the more convenient form:

$M_{\mathrm{NSC}}=5.36 \cdot 10^{6} \mathrm{M}_{\odot}\left(\frac{2 \mathrm{kpc}}{R_{\mathrm{Sph}}}\right)^{2}\left(\frac{250 \mathrm{~km} \mathrm{~s}^{-1}}{\sigma}\right)\left(\frac{\bar{m}_{\mathrm{GC}}}{10^{6} \mathrm{M}_{\odot}}\right)^{2}\left(\frac{N_{\mathrm{GC}}}{10^{2}}\right)$

where we have assumed a galaxy age of 10 Gyrs, $f_{g}=0.16$, and $\ln \Lambda=10$.

\subsection{Comparison with Observations}

We proceed to compare the predictions of the two formation scenarios discussed in the previous sections to the observed NSC masses, $M_{\mathrm{NSC}}^{\mathrm{obs}}$.

For the in-situ star formation model, the predicted NSC masses, $M_{\mathrm{NSC}}^{\mathrm{pred}}$, as calculated from Equation (6) are plotted against the observations in Figure 4 A weighted leastsquares fit yields the relation

$\log \left(\frac{M_{\mathrm{NSC}}^{\mathrm{obs}}}{10^{7.6} \mathrm{M}_{\odot}}\right)=(0.68 \pm 0.07) \log \left(\frac{M_{\mathrm{NSC}}^{\mathrm{pred}}}{10^{9.7} \mathrm{M}_{\odot}}\right)-(0.24 \pm 0.09)$,

The correlation is rather strong $\left(r_{s}=0.75, p_{s}=2.34 \cdot 10^{-10}\right)$, and has a scatter of 0.54 dex. However, the offset between the observed and predicted values suggests that the in-situ formation model over-predicts the observations by roughly two orders of magnitude across the galaxy sample. Given that secular evolution processes such as additional infall of cold molecular gas (e.g. Schinnerer et al. 2006, 2007) and/or young star clusters may well have added to the observed present-day NSC masses, and that all mechanisms for mass loss, such as the ejection of stars due to dynamical encounters, appear negligible in NSCs (e.g. Spitzer 1987; Heggie \& Hut 2003; Valtonen \& Karttunen 2006) this appears to present a severe challenge for the McLaughlin, King \& Nayakshin (2006) model.

Figure 5 makes the corresponding comparison for the GC infall model. Here, the predicted NSC masses were calculated using Equation 12 The solid line in Figure 5 is the best fit linear relation between the two quantities, described by

$\log \left(\frac{M_{\mathrm{NSC}}^{\mathrm{obs}}}{10^{7.6} \mathrm{M}_{\odot}}\right)=(0.42 \pm 0.26) \log \left(\frac{M_{\mathrm{NSC}}^{\mathrm{pred}}}{10^{6.0} \mathrm{M}_{\odot}}\right)-(0.74 \pm 0.13)$

The best-fit correlation has a slope that is marginally consistent with zero, has a large scatter of $0.80 \mathrm{dex}$, and is statistically weak $\left(r_{s}=0.49, p_{s}=5.24 \cdot 10^{-4}\right)$. Moreover, the $\mathrm{y}$-intercept suggests that the GC infall model under-predicts the observed NSC masses by about an order of magnitude across the galaxy sample. Adding to this problem is the fact that, as pointed out by Agarwal \& Milosavljevic (2011), many GCs may be disrupted before reaching the nucleus,

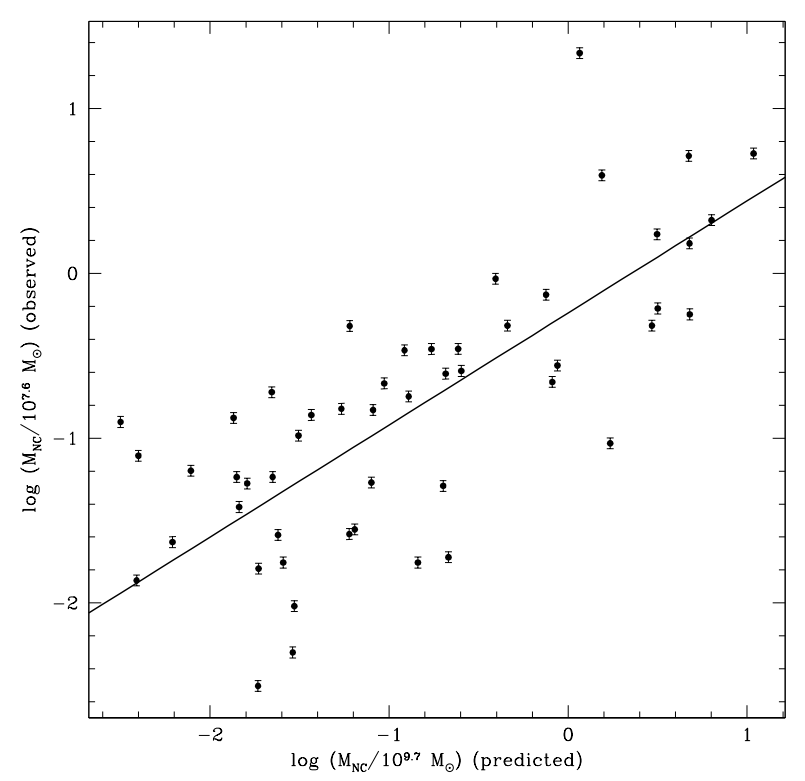

Figure 4. Observed versus predicted NSC masses for the in situ star formation model, with the latter calculated using Equation 6 The solid line shows a weighted least-squares fit to the data according to Equation 14 Error bars are described as in Figure 1

which would result in an even smaller NSC mass. We conclude that the GC infall model provides a poor description of the observations.

The multi-variate analysis presented in $\$ 3.3$ offers an additional test of the in situ star formation and GC infall models. In particular, from Equation [6, the in situ star formation model predicts $\alpha=2, \beta=-2$, and $\delta=0$, whereas, from Equation 12 the GC infall model predicts $\alpha=-1 / 2$, $\beta=-3 / 2$, and $\delta=1$. Therefore, if the various combinations of these values for $\alpha, \beta$, and $\delta$ for one of our competing formation scenarios are found to lie within the joint confidence intervals in every 2-dimensional projection of this 3-dimensional parameter space, this could be interpreted as evidence that the data are consistent with that particular formation scenario.

The $1 \sigma$ and $2 \sigma$ joint confidence intervals for all three projections of our 3-dimensional parameter space are shown in Figure 3. The theoretically predicted relations are shown by solid triangles and circles for the in situ star formation and GC infall models, respectively, as described in the figure caption. The precise values for the power-law indices corresponding to both the in situ and GC infall models do not coincide with even the $2 \sigma$ confidence intervals in any of the three projections.

\section{DISCUSSION}

Our results suggest that $M_{\mathrm{NSC}}$ scales linearly with luminous spheroid mass $M_{\mathrm{Sph}}$. We also find a dependence of the form $M_{\mathrm{NSC}} \propto \sigma^{2.73 \pm 0.29}$ when using velocity dispersions estimated via the virial theorem. This appears to support the conclusion of Graham (2012) that the observed $M_{\mathrm{NSC}}-\sigma$ 


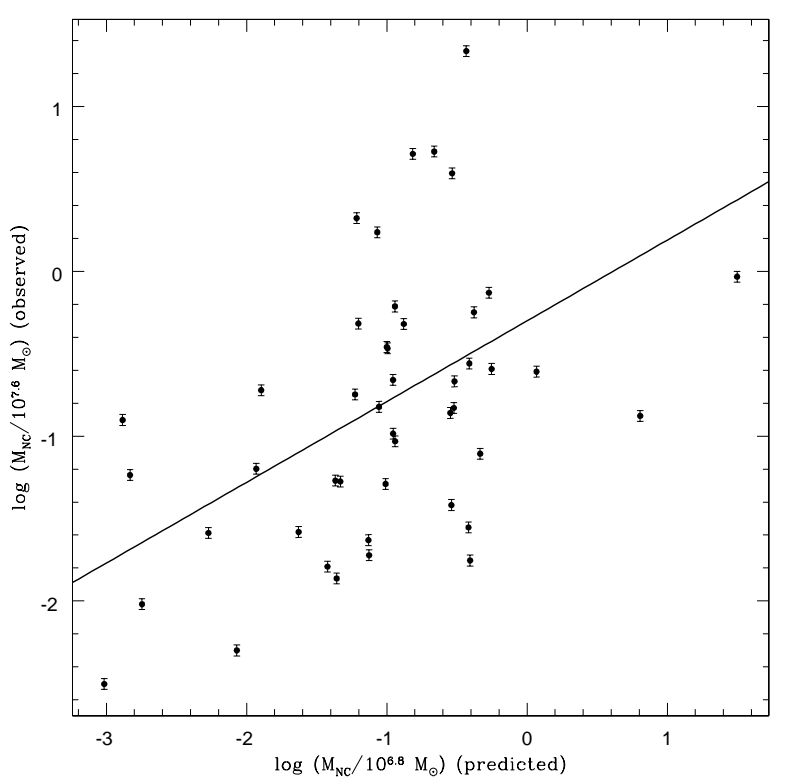

Figure 5. Observed versus predicted NSC masses for the GC infall model, with the latter calculated using Equation 12 The solid line shows a weighted least-squares fit to the data according to Equation 15 Error bars are described as in Figure 1

relation is not an extension of the observed $M_{\mathrm{SMBH}}-\sigma$ relation. Our results thus add further evidence to the notion that NSCs and SMBHs may not share a common origin, and that, instead, different formation mechanisms may be responsible for the two incarnations of a central massive object. We note that we find a slightly stronger correlation for the $\sigma-M_{\mathrm{NSC}}$ relation than we do for the $M_{\mathrm{Sph}}-M_{\mathrm{NSC}}$ relation. This raises the question whether perhaps a more fundamental relation is that between the velocity dispersion of the host spheroid and that of the NSC.

Such a relation could be explained if the NSCs are in thermal equilibrium with their host spheroids, possibly because galaxies tend to evolve towards (or be born in) such a state. To test this, we again used the virial theorem to calculate velocity dispersions also for all NSCs in the galaxy sample, using their effective radii measured by Cote et al. (2006), and listed in Column 9 of Table 1] Figure6 shows that both quantities are indeed correlated, with a linear fit yielding

$\log \left(\frac{\sigma_{\mathrm{NC}}}{102 \mathrm{~km} \mathrm{~s}^{-1}}\right)=(1.27 \pm 0.09) \log \left(\frac{\sigma}{128 \mathrm{~km} \mathrm{~s}^{-1}}\right)-(0.01 \pm 0.02)$

The correlation is rather strong $\left(r_{s}=0.73\right)$, and has a smaller scatter $(0.24 \mathrm{dex})$ around the best linear fit than either the $M_{\mathrm{Sph}}-M_{\mathrm{NSC}}$ or the $\sigma-M_{\mathrm{NSC}}$ relation. This supports the idea that the the dynamical evolution of NSCs is significantly affected by heat input from the surrounding galaxy (e.g. Merritt 2009). If indeed the present-day NSCs have reached an energy balance with their host spheroids, it implies that the time-scale for energy exchange between them is short compared to the age of the galaxy. This suggests that the present-day properties of NSCs do not necessarily reflect those at the time of their formation, since there

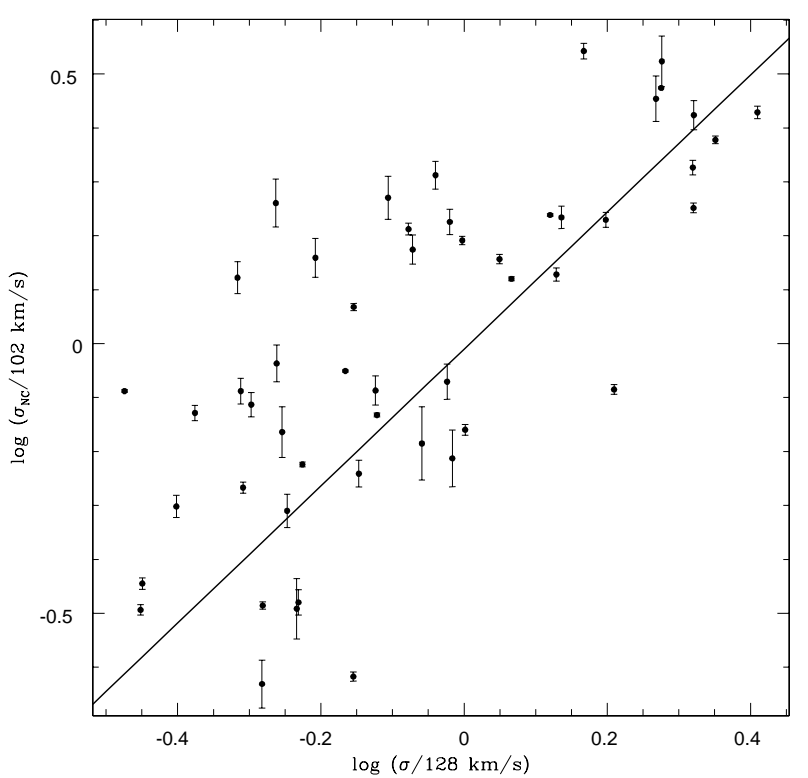

Figure 6. Comparison of the stellar velocity dispersion for NSC and host spheroid. The solid line shows a weighted least-squares fit to the data according to Equation16 Error bars were calculated using the $0.041 \mathrm{mag}$ and 0.007 arcsecond uncertainties quoted by Cote et al. (2006) for the NSC apparent magnitudes and radii, respectively.

was ample time for them to be modified by the presence of the surrounding spheroid.

The masses we have used for our analysis are derived from photometry, and therefore measure only the stellar mass of the galaxy. Measured velocity dispersions, on the other hand, provide a better proxy for the total mass of the spheroid, including the dark matter halo. It would therefore be most useful to perform a similar analysis using measured velocity dispersions once they become available. Nevertheless, because of the arguments presented in Section 3.3. our results have cast some doubt on the assumption that the baryonic mass fraction $f_{g}$ is the same for all early-type galaxies and/or that the dark matter has the same radial profile as the luminous matter.

We have also compared the observed present-day NSC masses to theoretical predictions from two proposed models for NSC formation, namely in-situ star formation regulated by momentum feedback and the successive infall of star clusters due to dynamical friction. As discussed in \$4.3 both models appear to have difficulties in predicting the observations.

The in-situ model predicts NSC masses that exceed the observed ones by about two orders of magnitude. Since the NSC may well have grown through secular processes throughout the life of the galaxy, this is probably only a lower limit for the true difference. The discrepancy may be somewhat reduced if one assumes that the host galaxy was significantly less massive and/or more extended at the time of NSC formation than it is today. In this case, Equation 6 would yield lower NSC masses. For example, if the NSC host was half as massive and twice as extended when the NSC formed, the resulting NSC mass should be $\sim 16$ times lower. 
On the other hand, in this scenario it is difficult to explain why the $M_{\mathrm{NSC}}-\sigma$ relation does not seem to have the predicted slope of $\beta=4$ (Graham 2012).

The GC infall model, on the other hand, systematically under-predicts the observed NSC masses by about an order of magnitude across the galaxy sample. This could, at least partly, be rectified by adopting a larger value for the average GC mass. Indeed, the most massive GCs are most responsive to dynamical friction, and therefore are most likely to have spiraled into the nucleus by today. Using the present-day average GC mass as an estimate for the average mass of the GCs that have merged onto the NSC in Equation 12 may therefore provide too low an estimate. Indeed, most NSC masses can be reproduced if 10 very massive GCs $\left(\gtrsim 10^{6} \mathrm{M}_{\odot}\right)$ had fallen in via dynamical friction. With the data at hand, we cannot rule out this possibility.

In summary, both models appear to have difficulties in explaining the observed NSC masses. Of course, this does not imply that neither in-situ star formation nor GC infall has occurred, but it suggests that either model by itself provides an over-simplified description of NSC formation.

\section{SUMMARY}

In this paper, we have performed a statistical analysis to study the observed $M_{\mathrm{NSC}}-M_{\mathrm{Sph}}$ and inferred $M_{\mathrm{NSC}}-\sigma$ relations using a large sample of 51 nucleated early-type galaxies taken from the ACSVCS. We used luminous galaxy masses, and calculated velocity dispersions found by assuming virial equilibrium applies for our sample of galaxies. We confirm a linear relationship between NSC mass and luminous host spheroid mass reported by previous studies, but infer a shallower slope of the $M_{\mathrm{NSC}}-\sigma$ relation than reported in Ferrarese et al. (2006a).

Our results support a recent claim (Graham 2012) that NSCs do not obey the same $M-\sigma$ relation as do SMBHs, adhering instead to a shallower dependence. Specifically, we found $M_{\mathrm{NSC}} \propto \sigma^{2.73 \pm 0.29}$. This appears to cast doubt on the notion that NSCs and SMBHs share a common evolutionary path, i.e. that they are merely different incarnations of "central massive object". We speculate that the discrepancy between our results and those of Ferrarese et al. (2006a), who found an $M_{\mathrm{NSC}}-\sigma$ relation closer to $M_{\mathrm{NSC}} \propto \sigma^{4}$ using observed velocity dispersions, can perhaps be explained if the baryonic mass fraction is not the same for all earlytype galaxies, or follows a different radial profile than the luminous matter.

Finally, we have compared the observed present-day NSC masses to first-order predictions of two proposed NSC formation scenarios, namely GC infall and feedbackregulated in-situ formation. We find that neither scenario can provide a reasonable agreement with the observations.

\section{ACKNOWLEDGMENTS}

We would like to thank Elizabeth Wehner for useful suggestions that improved the quality of our paper, and Laura Ferrarese for helpful discussions regarding the measured velocity dispersion of the ACSVCS galaxies.

\section{REFERENCES}

Agarwal M., Milosavljevic M. 2011, ApJ, 729, 35

Antonini F., Capuzzo-Dolcetta R., Mastrobuono-Battisti A., Merritt D. 2012, ApJ, 750, 111

Balcells M., Graham A. W., Domínguez-Palmero L., Peletier R. F. 2003, ApJ, 582, 79

Balcells M., Graham A. W., Peletier R. F. 2007, ApJ, 665, 1104

Bell E. F., McIntosh D. H., Katz N., Weinberg M. D. 2003, ApJS, 149, 289

Binney J., Tremaine S., 1987, Galactic Dynamics (Princeton: Princeton University Press)

Böker T., Laine S., van der Marel R. P., Sarzi M., Rix H.-W., Ho L. C., Shields J. C. 2002, AJ, 123, 1389

Böker T., Sarzi M., McLaughlin D. E., van der Marel R. P., Rix H.-W., Ho L. C., Shields J. C. 2004, AJ, 127, 105 Cappellari M., Bacon R., Bureau M., Damen M. C., Davies R. L., de Zeeuw P. T., Emsellem E., Falcón-Barroso J., et al. 2006, MNRAS, 366, 1126

Capuzzo-Dolcetta R., Miocchi P. 2008, MNRAS, 388, 69

Carollo C. M., Stiavelli M., Mack J. 1998, AJ, 116, 68

Cote P., Blakeslee J. P., Ferrarese L., Jordan A., Mei S., Merritt D., Milosavljevic M., Peng E. W., et al. 2004, ApJS, 153, 223

Cote P., Piatek S., Ferrarese L., Jordan A., Merritt D., Peng E. W., Hasegan M., Blakeslee J. P., et al. 2006, ApJS, 165, 57

Ferrarese L., Merritt D. 2000, ApJ, 539, L9

Ferrarese L., Cote P., Dalla Bonta, E., Peng E. W., Merritt D., Jordan A., Blakeslee J. P., Hasegan, M., et al. 2006a, ApJ, 644, L21

Ferrarese L., Cote P., Jordan A., Peng E. W., Blakeslee J. P., Piatek S., Mei S., Merritt D., et al. 2006b, ApJS, 164, 334

Gallo E., Treu T., Marshall P. J., Woo J.-H., Leipski C., Antonucci R. 2010, ApJ, 714, 25

Gebhardt K., Bender R., Bower G., Dressler A., Faber S. M., Filippenko A. V., Green R., Grillmair C., Ho L. C., Kormendy J., Lauer T. R., Magorrian J., Pinkney J., Richstone D., Tremaine S. 2000, ApJ, 539, L13

Gonzalez Delgado R. M., Perez E., Cid Fernandes R., Schmitt H. 2008, AJ, 135, 747

Graham A. W., Guzman R. 2003, AJ, 125, 2936

Graham A. W., Driver S. P. 2007, ApJ, 655, 77

Graham A. W., Spitler L. R. 2009, MNRAS, 397, 2148

Graham A. W. 2012, MNRAS, 422, 1586

Häring N., Rix H.-W. 2004, ApJ, 604, L89

Hartmann M., Debattista V. P., Seth A., Cappellari M., Quinn T. R. 2011, MNRAS, 418, 2697

Heggie D. C., Hut P. 2003, The Gravitational Million-Body Problem: A Multidisciplinary Approach to Star Cluster Dynamics (Cambridge: Cambridge University Press)

King A. R. 2003, ApJL, 596, L27

King A. R. 2005, ApJL, 635, L121

Leigh N. W., Sills A., Knigge C. 2011, MNRAS, 415, 377

Leitherer C., Robert C., Drissen L. 1992, ApJ, 401, 596

Lotz J. M., Miller B. W., Ferguson H. C. 2004, ApJ, 613, 262

McLaughlin D. E., King A. R., Nayakshin S. 2006, ApJL, 650, L37

Merritt D. 2009, ApJ, 694, 959 
Nayakshin S., Wilkinson M. I., King A. 2009, MNRAS, 398, 54

Neumayer, N. \& Walcher, C. J. 2012, Advances in Astronomy, 2012, 15

Peng E. W., Jordan A., Cote P., Takamiya M., West M., Blakeslee J. P., Chen C.-W., Ferrarese L., et al. 2008, ApJ, 681, 197

Quinlan G. D., Shapiro S. L. 1990, ApJ, 356, 483

Rossa J., van der Marel R. P., Böker T., Gerssen J., Ho L. C., Rix H.-W., Shields J. C., Walcher C.-J. 2006, AJ, 132, 1074

Satyapal S., Vega D., Dudik R. P., Abel N. P., \& Heckman T. 2008, ApJ, 677, 926

Satyapal S., Böker T., Mcalpine W., Gliozzi M., Abel N. P., \& Heckman T. 2009, ApJ, 704, 439

Schinnerer, E., Böker, T., Emsellem, E., \& Lisenfeld, U. 2006, ApJ, 649, 181

Schinnerer, E., Böker, T., Emsellem, E., \& Downes, D. 2007, A\&A, 462, L27

Seth A., Agueros M., Lee D., Basu-Zych A. 2008, ApJ, 678, 116

Spergel D. N., Verde L., Peiris H. V., Komatsu E., Nolta M. R., Bennett C. L., Halpern M., Hinshaw G., et al. 2003, ApJS, 148, 175

Spitzer L. Jr. 1987, Dynamical Evolution of Globular Clusters (Princeton, NJ: Princeton Univ. Press)

Tremaine S. D., Ostriker J. P., Spitzer L. Jr. 1975, ApJ, 196, 407

Valtonen M., Karttunen H. 2006, The Three-Body Problem (Cambridge: Cambridge University Press)

Verbunt F., Pooley D., Bassa C. 2007, in Dynamical Evolution of Dense Stellar Systems, IAU Symp. 246, ed. E. Vesperini (Dordrecht: Reidel), 1

Walcher C. J., van der Marel R. P., McLaughlin D., Rix H.-W., Böker T., Häring N., Ho L. C., Sarzi M., et al. 2005, ApJ, 618, 237

Walcher C. J., Böker T., Charlot S., Ho L. C., Rix H.-W., Rossa J., Shields J. C., van der Marel R. P. 2006, ApJ, 649,692

Wehner E. H., Harris W. E. 2006, ApJL, 644, L17

This paper has been typeset from a $\mathrm{T}_{\mathrm{E}} \mathrm{X} / \mathrm{L} \mathrm{AT}_{\mathrm{E}} \mathrm{X}$ file prepared by the author. 